PTH-74 CO-PRODUCTION OF PATIENT INFORMATION LEAFLET WITH NORTHERN IRELAND BARRETT'S OESOPHAGUS PUBLIC AND PATIENT INVOLVEMENT GROUP

${ }^{1}$ Victoria Cairnduff, ${ }^{2}$ Ariana Axiaq, ${ }^{3}$ Rhonda Campbell, ${ }^{3}$ John Clarke, ${ }^{3}$ Mark Kelly, ${ }^{3}$ Rosemary McAnerney, ${ }^{4}$ Damian T McManus, ${ }^{5}$ Richard C Turkington, ${ }^{6}$ Brian T Johnston, 1,5 Helen G Coleman. 'Centre for Public Health, Queen's University Belfast, Belfast, UKi

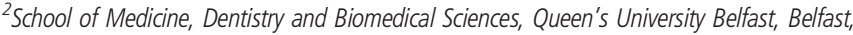
UK; ${ }^{3}$ Northern Ireland Barrett's oesophagus research Public and Patient Involvement group, Belfast, UK; ${ }^{4}$ Department of Pathology, Belfast Health and Social Care Trust, Belfast, UK ${ }^{5}$ Patrick G Johnston Centre for Cancer Research, Queen's University, Belfast, UK; ${ }^{6}$ Department of Gastroenterology, Belfast Health and Social Care Trust, Belfast, UK

\subsection{6/gutjnl-2021-BSG.253}

Introduction The Northern Ireland Barrett's oesophagus research Public and Patient Involvement (PPI) group identified an area of unmet need for standardisation of the information provided to patients at the time of their Barrett's oesophagus diagnosis. This study aimed to co-produce a patient information leaflet as an evidence-based resource for Barrett's oesophagus patients.

Methods The PPI group first initiated the co-production of the leaflet in May 2020, providing initial ideas based on patient experience and information needs. A scientific literature review in Pubmed was conducted to identify reported information needs of Barrett's oesophagus patients. The leaflet has received two rounds of iterative feedback at 1.5 hour PPI group meetings and clinical input from Consultants in the areas of Gastroenterology, Pathology and Oncology.

Results The review of the literature and PPI group feedback identified several important areas for inclusion in the leaflet:

- What is Barrett's oesophagus?

- How is Barrett's oesophagus is diagnosed?

Risk factors associated with Barrett's oesophagus development

- Symptoms associated with Barrett's oesophagus and ways they are managed,

- Treatment plans including endoscopic surveillance and medications prescribed

- Quantifying the risk of progression to oesophageal adenocarcinoma

- Recommended online information sources

The PPI group highlighted the need for clear images and diagrams alongside the written text to aid patient understanding of Barrett's oesophagus with the clinicians emphasising the need for the leaflet to be short and therefore good optimisation of space. Balancing risk communication of a pre-malignant condition with a relatively low lifetime risk of cancer progression, with recommendations to reduce this risk, was a key focus of discussions and feedback.

Conclusion A four A5 page evidenced-based leaflet on Barrett's oesophagus has been developed, aiming to address previously unmet information needs. The next phase will evaluate the leaflet in other Barrett's oesophagus patients and key stakeholders involved in their care. Future plans include the development of an online patient resource.

\section{PTH-75 THE UTILITY OF PLAIN CHEST RADIOGRAPHS TO DETECT PERFORATION AFTER ENDOSCOPIC STENTING FOR OESOPHAGEAL CANCER}

Daniel Maggs*, Hannah Thould, Ben Colleypriest. Royal United Hospital, Bath, UK

10.1136/gutjnl-2021-BSG.254
Introduction In line with BSG guidelines (2011), endoscopic placement of self-expanding stents has become standard practice for the palliative management of stenosing oesophageal tumours. Procedural protocol varies between centres. Locally a plain chest radiograph (CXR) is performed 2 hours post stenting to check expansion and for signs of oesophageal perforation prior to commencing clear fluids orally. We aimed to assess compliance with this protocol and evaluate the use of CXR to exclude oesophageal perforation.

Methods Consecutive day case and inpatient oesophageal stent procedures were identified from clinical coding between January 2017 and December 2020. Electronic patient records only were interrogated. Data was collected on the use of balloon dilatation, CXR reports, alternative or additional imaging, presence of perforation, and survival post-stent. Basic statistics were performed using Microsoft Excel.

Results Seventy patients were identified with a median age of 77 years. There were 80 procedures performed during the study period. Ten patients required redo oesophageal stenting. The majority had a day case procedure (58\%). Balloon dilatation was performed prior to stenting in $39 \%$ of procedures. Median survival post-stenting was 78 days (IQR 32-174).

A CXR was performed post-stenting in only $89 \%$ of cases despite being part of post-procedure protocol. When a CXR was not performed a there was no rationale documented in the electronic records. 13 patients (16.2\%) required further imaging, with either computed tomography (CT) or watersoluble contrast swallow, to exclude oesophageal perforation.

Oesophageal perforation was identified in 3 patients $(3.75 \%)$ post procedure. A CXR was performed in all 3 cases however only one revealed evidence of perforation. One patient had a perforation identified during endoscopy, following a balloon dilatation. The post procedure CXR demonstrated pneumoperitoneum; however subsequent water- soluble contrast swallow showed no leak. Unfortunately, the patient died on day 7 of admission however it was not clear if this was a result of oesophageal perforation. The remaining 2 cases of oesophageal perforation were only identified on CT performed due to high clinical suspicion. Both patients were discharged without further complications. All 3 cases were managed conservatively.

Conclusions We have shown that there was not full compliance with local protocol and that CXR was not solely relied upon for diagnosing oesophageal perforation. CXR alone is not sensitive enough to confidently exclude perforation when there is significant clinical concern. It should also be noted that, on the day of procedure, radiology reported CXR's are rarely available leaving more junior staff to interpret the images. An argument could be made for replacing routine post-procedure CXR with clinical criteria to guide use of more sensitive imaging modalities such as CT.

\section{PTH-76 USE OF ORODISPERSIBLE BUDESONIDE IN THE TREATMENT OF EOSINOPHILIC OESOPHAGITIS: A SINGLE-CENTRE AUDIT}

${ }^{1}$ Bo Wang * , Laura Blackmore, 'Esha Sharma, 'Vernise Daji, ${ }^{1}$ Terry Wong, ${ }^{1,2}$ Sebastian Zeki, ${ }^{1,2}$ Jason Dunn. 'Guy's and St Thomas' NHS Foundation Trust, London, UK; ${ }^{2}$ King's College London, London, UK

10.1136/gutjnl-2021-BSG.255

Introduction Eosinophilic oesophagitis (EoE) is a chronic inflammatory condition of the oesophagus. The diagnosis is 
made in individuals presenting with dysphagia and/or food bolus impaction, based on typical endoscopic findings (expressed as EREFS score determining the severity of 5 endoscopic findings: oedema, rings, exudates, furrows and strictures) and confirmative histology (>15 eosinophils per highpower field). Treatments include unlicensed swallowed fluticasone via a metered-dose inhaler, proton-pump inhibitors (PPI) and elimination diets, though clinico-histological remission is variable. Orodispersible budesonide (Jorveza) is the first licensed therapy for EoE and has recently been approved by NICE for induction treatment in the UK. This is an audit of the first six months' experience of its use in our centre.

Methods Pharmacy prescription records were used to identify patients who had been prescribed orodispersible budesonide between September 2020 and March 2021. Case note review was performed to document treatment history, baseline endoscopic and histological findings, clinical and endoscopic response, follow-up and adverse effects.

Results 27 patients were identified; 78\% were male, with a mean age of 45 years (range 23-74). 85\% were symptomatic with dysphagia and $44 \%$ had a history of food bolus impaction. $93 \%$ of patients had failed medical therapy before starting orodispersible budesonide and $63 \%$ had failed an elimination diet. All patients had a baseline endoscopy prior to starting treatment, with a mode EREFS score of 3 . The majority of patients $(78 \%)$ were treated with a 6 week course and the remainder received 12 weeks, with 6 patients $(22 \%)$ going on to a maintenance dose.

$94 \%$ of patients had a clinical review within 12 weeks of the original prescription. Overall, $86 \%$ achieved symptomatic remission. $56 \%$ of patients had a follow-up endoscopy after at least 6 weeks of treatment, with some impact from reduction of endoscopy services during the second wave of the COVID19 pandemic. Of those who had a follow-up endoscopy, 50\% achieved endoscopic remission, as defined by an EREFS of 0 . $61 \%$ achieved histological remission, as defined by an eosinophil count of $<15$ per high-power field (hpf). The mean drop in peak eosinophil count was 58 per hpf (range 4-117).

Three patients reported adverse effects with two reporting new onset gastro-oesophageal reflux symptoms and one developing acne.

Conclusions Our tertiary single-centre experience demonstrates good adherence with NICE guidance regarding use of orodispersible budesonide in EoE. Clinical, endoscopic and histological remission is achieved in the majority of cases, in line with published evidence supporting its use.

\section{PTH-77 BIOPSIES FOR GRADE D OESOPHAGITIS; DO WE NEED THEM?}

Tamara Glaister*, Julia F Simons, Suneil A Raju, John M Hebden. Academic Unit of Gastroenterology, Royal Hallamshire Hospital, Sheffield, UK

\subsection{6/gutjnl-2021-BSG.256}

Introduction British Society of Gastroenterology guidelines state all patients with Los Angeles (LA) grade D oesophagitis should be biopsied and re-evaluated at 6 weeks to exclude dysplasia and malignancy. There is a paucity of data showing outcomes. We present the largest data on the clinical use of biopsies in patients with grade D oesophagitis.

Methods All patients with LA grade D oesophagitis were identified between January 2018 and December 2019 in a tertiary teaching hospital. Further data was collected from case notes and on subsequent endoscopies. Patient outcomes were followed up until May 2021.

Results In total, 132 patients were identified with grade D oesophagitis on index gastroscopy [median age 69 years, (IQR 55 - 78 years), 59\% male]. Indications for index gastroscopy were suspected gastrointestinal bleed (37\%), dysphagia (21\%), anaemia (15\%) and nausea (14\%).

Oesophageal biopsies were taken in $21 \%$ of index gastroscopies median 3 (IQR 2-5). Biopsies identified oesophageal ulceration (37\%), reflux oesophagitis (26\%), Barrett's (19\%) and erosive oesophagitis (15\%). No dysplasia or carcinoma was identified histologically or endoscopically.

Repeat gastroscopy was performed in $57 \%$ of patients at median 55 days (IQR 42-70 days) and 16\% reported on-going grade D oesophagitis. Biopsies were taken during repeat gastroscopies in $33 \%$ of cases. No dysplasia or carcinomas were identified, however $23 \%$ of patients were found to have Barrett's oesophagus which was not previously identified. During the follow-up period no oesophageal cancer was identified.

Conclusions No dysplasia or carcinoma was discovered at index or follow-up gastroscopy, however, repeat gastroscopies are still required to exclude other diagnoses. Adherence to biopsy guidelines is low, as is follow-up gastroscopy for patients with LA grade D oesophagitis though during the follow-up period, no oesophageal cancers were identified.

\section{PTH-78 COMPUTATIONAL COLOUR CONTRAST-ENHANCEMENT IMPROVES ENDOSCOPIC VISIBILITY OF OESOPHAGEAL SQUAMOUS DYSPLASIA AND DETECTION IN AI-BASED SYSTEM}

${ }^{1}$ Xiaohong Gao, ${ }^{2}$ Stephen Taylor, ${ }^{3}$ Prof Wei Pang, ${ }^{4}$ Rui Hui, ${ }^{5}$ Prof Xin Lu, ${ }^{6}$ Investigators TGU Oxford, ${ }^{6}$ Barbara Braden*. 'Middlesex University, London, UK; ${ }^{2}$ University of Oxford, Oxford, UK; ${ }^{3}$ University of Heriot-Watt, Edinburgh, UK; ${ }^{4}$ Beijing Navy General Hospital, Bejing, China; ${ }^{5}$ Ludwig Institute, University of Oxford, Oxford, UK; ${ }^{6}$ Translational Gastroenterology Unit, Oxford, Oxford, UK

\subsection{6/gutjnl-2021-BSG.257}

Introduction White light (WLI) endoscopy often misses precancerous oesophageal changes in squamous epithelium due to their only subtle visual differences to the surrounding normal mucosa. This paper aims to exploit computational enhancement of colour contrast from human perception point of view to increase the visibility of squamous dysplasia and to optimise a deep learning-based decision support system.

Methods 905 WLI images from 277 patients including histologically confirmed low grade (LGD), high grade dysplasia (HGD) or intramucosal squamous cancer (SCC) together with their corresponding narrow band (NBI) were evaluated. By applying the Commission Internationale de L'Eclairage (CIE) colour appearance model CIECAM02 to represent an image using human perceptual attribute correlates, each pixel of an image was enhanced and converted back to RGB values to display contrasted images. Colour differences between dysplastic regions and normal surrounding mucosa were measured in the original images and in the contrast-enhanced images. These contrast-enhanced images were also embedded to train a deep learning-based detection system that was tested in an external independent cohort of 112 patients (70 normal).

Results Through the modelling of colour appearance and contrast enhancement based on human colour vision, a real-time endoscopy decision support system was developed that works 